\title{
FKBP10/FKBP65 expression in high-grade ovarian serous carcinoma and its association with patient outcome
}

\author{
MICHAEL C.J. QUINN ${ }^{1 *}$, PAULINA M. WOJNAROWICZ ${ }^{2 *}$, AMY PICKETT $^{3}$, DIANE M. PROVENCHER ${ }^{1,4,5}$, \\ ANNE-MARIE MES-MASSON ${ }^{1,6}$, ELAINE C. DAVIS $^{3}$ and PATRICIA N. TONIN ${ }^{2,7,8}$ \\ ${ }^{1}$ Research Centre of the University of Montreal Hospital Centre/Montreal Cancer Institute; \\ ${ }^{2}$ Department of Human Genetics, McGill University; ${ }^{3}$ Faculty of Medicine, Department of Anatomy \\ and Cell Biology, McGill University; ${ }^{4}$ Division of Gynecologic Oncology, University of Montreal; \\ Departments of ${ }^{5}$ Obstetrics and Gynecology and ${ }^{6}$ Medicine, University of Montreal; \\ ${ }^{7}$ The Research Institute of the McGill University Health Centre; ${ }^{8}$ Department of Medicine, \\ McGill University, Montreal, QC, Canada
}

Received October 31, 2012; Accepted December 5, 2012

DOI: 10.3892/ijo.2013.1797

\begin{abstract}
The frequent loss of chromosome 17 in epithelial ovarian carcinomas (EOC), particularly high-grade serous carcinomas (HGSC), has been attributed to the disruption of TP53 (at 17p13.1) and other chromosome 17 genes suspected to play a role in tumour suppressor pathways. In a transcriptome analysis of HGSC, we showed underexpression of a number of chromosome 17 genes, which included FKBP10 (at 17q21.1) and collagen $I \alpha 1$ (COL1A1; at 17q21.33). FKBP10 codes for the immunophilin FKBP65 and is suspected to act as a chaperone for COL1A1. We have investigated FKBP10 (gene) and FKBP65 (protein) expression in HGSC samples and EOC cell lines that differ in their tumourigenic potential. COL1A1 expression was also investigated given the purported function of FKBP65. RT-PCR analysis verified underexpression of FKBP10 and COLIAl in HGSCs $(\mathrm{n}=14)$ and six tumourigenic EOC cell lines, relative to normal ovarian surface epithelial cells and a non-tumourigenic EOC cell line. Immunohistochemistry analyses of 196 HGSC samples using tissue microarrays revealed variable staining intensities in the epithelial tumour component where only $7.8 \%$ and $1.0 \%$ of samples stained intensely for FKBP65 and COL1A1, respectively. Variable staining intensities were also observed for the stromal component where $23.6 \%$ and $24.1 \%$ stained intensely for FKBP65 and COL1A1, respec-
\end{abstract}

Correspondence to: Dr Patricia N. Tonin, Medical Genetics, Room L10-132, Montreal General Hospital, 1650 Cedar Avenue, Montreal, QC H3G 1A4, Canada

E-mail: patricia.tonin@mcgill.ca

*Contributed equally

Key words: high-grade ovarian serous carcinoma, FKBP10, FKBP65, COL1A1, chromosome 17, gene expression, tissue microarray, immunohistochemistry tively. There was no significant correlation of staining intensity of either protein with disease stage. Staining of FKBP65 was clearly visible in normal epithelial cells of the ovarian surface and fallopian tube. There was a significant correlation between absence of FKBP65 staining in the epithelial cell component of the tumour and prolonged overall survival $(\mathrm{p}<0.001)$. Our results suggest that underexpression of FKBP65 protein is characteristic of HGSCs and that this expression profile may be linked to molecular pathways associated with an unfavourable outcome in cancer patients.

\section{Introduction}

Loss of heterozygosity of chromosome 17 is a frequent occurrence in epithelial ovarian cancer (EOC) and particularly in high-grade serous carcinomas (HGSC), which is one of the most common histotypes of EOCs (1-6). This has been largely attributed to the inactivation of the tumour suppressor gene TP53, which is located at $17 \mathrm{p} 13.1$, as it is the most frequently mutated gene in HGSCs, however, other genes involved in tumour suppressor pathways have been proposed (1-4,7-14). For example, our group recently identified 158 underexpressed chromosome 17 genes in a transcriptome analysis of HGSCs, which included $F K B P 10$, a gene that maps to $17 \mathrm{q} 21.1$. Interestingly, $F K B P 10$ was among the genes upregulated in a genetically modified EOC cell line rendered non-tumourigenic as a consequence of an unique gene complementation assay involving chromosome transfer, and thus may be one of a number of genes transcriptionally reprogrammed as a consequence of tumour suppression $(15,16)$.

FKBP10 encodes FKBP65, a 65 kDa FK506 binding protein that is a member of the FKBP-type peptidyl-prolyl cis/trans isomerase family $(17,18)$. This protein localizes to the endoplasmic reticulum and acts as a molecular chaperone and binding partner of type I collagen (19-21). The interaction with type I collagen is interesting as $C O L 1 A 1$, which is a gene that maps to $17 \mathrm{q} 21.33$, was also found significantly underexpressed in our chromosome 17 transcriptome analyses of HGSCs (14). FKBP65 
is expressed in developing tissues and re-expressed in adult tissues following injury $(19,20)$. In the mouse, the only tissues that continue to express FKBP65 are reproductive tissues (ovary, uterus and mammary glands) during phases of growth and remodelling (E.C. Davis, unpublished data). Although somatic mutations inactivating $F K B P 10$ have not been identified, such as in recent reports of genome-wide exomic sequencing analyses of HGSCs by The Cancer Genome Atlas Research Network (5) and other cancer types (22), germline FKBP10 mutations have been described in association with autosomal-recessive osteogenesis imperfecta and Bruck syndrome, which are connective tissue disorders characterized by defects in type I collagen $(23,24)$. Though epithelial malignancies in patients with osteogenesis imperfecta are rare, there is at least one case report of a 32-year-old who developed a low-grade serous ovarian carcinoma with stage IIIb disease (25).

The localization of FKBPIO to chromosome 17 and its interesting expression profile in the murine adult normal ovary, HGSCs and our genetically modified EOC cell line, prompted our further investigation of this gene in ovarian cancer samples. In this study, we investigated the expression profile of $F K B P 10$ in HGSCs, and its expression in a set of well-characterized EOC cell lines that differ in their growth characteristics and tumourigenic potential. We also investigated protein expression by immunohistochemistry analysis of a tissue microarray and related the expression profile to patient outcome. COLIAI gene and protein expression was also investigated given its purported interaction with FKBP65.

\section{Materials and methods}

Tissue specimens, cell lines, and clinical information. The HGSCs, primary cultures of normal ovarian surface epithelial cells (NOSE), and EOC cell lines (OV90, TOV112D, TOV81D, TOV21G, TOV1946, OV1946 and TOV2223) examined for gene expression have been described previously $(1,14,26)$. Briefly, the HGSC samples were from chemotherapy naïve patients and the cell lines were derived from long-term passages of malignant ovarian ascites from an undifferentiated adenocarcinoma (OV-90), high-grade endometrioid adenocarcinoma (TOV112D), serous carcinomas (TOV81D, TOV1946, OV1946 and TOV2223) and a clear cell carcinoma (TOV21G), where TOV1946 and OV1946 were derived from malignant ovarian ascites (OV1946) or tumour (TOV1946) from the same patient.

The HGSC $(n=196)$ cases represented in the tissue array were derived from archival blocks of paraffin-embedded tissues samples as previously described (27). The tumour grade and disease stage (Table I) were designated according to the International Federation of Gynaecology and Obstetrics. Disease-free interval, defined as time to doubling of the upper normal limit of the serum cancer antigen marker CA-125 or the detection of a new lesion by ultrasound or CT-scan imaging, and overall survival, defined according to the Response Evaluation Criteria in Solid Tumors (28) were extracted from the Système d'Archivage des Données en Oncologie (Table I). Normal ovary and fallopian tube tissues used in immunohistochemistry analyses were retrieved from archival paraffin-embedded samples. Normal ovarian tissues were obtained from ovarectomy (age 40) and hysterectomy (age 45) cases due to a benign uterine tumour. The fallopian tube tissues were adjacent normal tissues collected from women diagnosed with serous ovarian cancer. All samples and related clinical information were obtained with informed written consent at the Centre Hospitalier de l' Universite de Montreal (CHUM) - Hôpital Notre Dame.

Gene expression analyses. Gene expression was assessed by semi-quantitative RT-PCR analysis using cDNA synthesized from total RNA prepared as previously described $(3,13,15,29)$. Primers were designed using Primer3 software (30) based on genomic structures of $F K B P 10$ and $C O L 1 A 1$ available from the March 2006 human reference sequence (NCBI Build 36.1/hg18) assembly (31) and alignment of reference sequences of each gene, NM_021939 and NM_000088.3, respectively. The FKBP10 forward primer is 5'-GTGGAACAAGGAAGA CACC-3', and the reverse primer is 5'-CTTCCTTCTCTCTCC AGGAC-3', yielding a 238 base pair product. The COL1A1 forward primer is 5'-GTGCTCCTGTATTGCTG-3', and the reverse primer is 5'-CTCGCTTTCCTTCCTCTC-3', yielding a 207 base pair product. The RT-PCR-based assays were performed essentially as previously described (32). RT-PCR of $18 S$ RNA was performed to assess RNA quality. Primer sequences for $18 S$ were reported previously (3).

Immunohistochemistry analysis of tissue arrays. FKBP65 and COL1A1 protein expression was assessed by immunohistochemistry analysis using a tissue array containing $0.6 \mathrm{~mm}$ cores derived from paraffin-embedded tissue blocks representing 196 HGSC cases selected based on a review of hematoxylin and eosin-stained slides prepared as described previously (27). The array also contained 11 normal fallopian tube samples. Immunohistochemistry analysis was performed on the tissue array and sections prepared from two normal ovary paraffin-embedded tissue blocks. Five-micron sections were mounted onto frosted plus slides, deparaffinized in Citrisolv (Fisher Scientific) for $90 \mathrm{~min}$ and then rehydrated in an ethanol gradient. Before primary antibody incubation, antigen retrieval was performed using $0.05 \%$ Tween- 20 in $10 \mathrm{mM}$ sodium citrate (pH 6.0) at $90^{\circ} \mathrm{C}$ for $20 \mathrm{~min}$. The slides were washed with $0.1 \%$ Triton X-100 in Tris-buffered saline (TBS) [0.5 M Tris, $1.5 \mathrm{M} \mathrm{NaCl},(\mathrm{pH} 7.4)]$. The slides were then washed and blocked with TBS containing $0.1 \%$ bovine serum albumin (TBS/BSA) for 3x10 min and then incubated with Ultra V Block (LabVision Corp., Fremont, CA, USA) for $7 \mathrm{~min}$. After washing again in TBS/BSA, the slides were incubated overnight at $4^{\circ} \mathrm{C}$ with either a polyclonal FKBP65 antibody, raised against a synthetic peptide to the C-terminus of the mouse FKBP65 (33), or a polyclonal type I collagen antibody (Calbiochem), diluted at 1:300 and 1:1000, respectively. Slides were incubated in Value Primary Antibody Enhancer (LabVision Corp.) for $20 \mathrm{~min}$ before incubation in AP Value Polymer (LabVision Corp.) for $30 \mathrm{~min}$ at room temperature. Staining was visualized using FastRed (LabVision Corp.) and slides were counterstained with hematoxylin for $30 \mathrm{sec}$. The tissue arrays were scanned with an Aperio ScanScope XT Digital Slide Scanner and images were viewed at high resolution using Aperio ImageScope Software version 11.02. Two observers examined the images independently and scored them based on staining intensity ranked as absent, low, moderate or high, for both the epithelial and stromal tissue components of each core. The inter-observer correlation coefficients were 0.792 for FKBP65 and 0.747 for 
Table I. Description of HGSC tissue array.

\begin{tabular}{|c|c|c|c|}
\hline Patient characteristic & All cases & $\mathrm{G} 2$ & G3 \\
\hline No. of cases & 196 & 30 & 166 \\
\hline Mean age of diagnosis, years (range) & $62(34-89)$ & $60(42-82)$ & $62(34-89)$ \\
\hline Stage I & 15 & 2 & 13 \\
\hline Stage II & 19 & 2 & 17 \\
\hline Stage III & 139 & 23 & 116 \\
\hline Stage IV & 23 & 3 & 20 \\
\hline Mean disease free interval, months (range) & $22(0-134)$ & $15(0-57)$ & $23(0-134)$ \\
\hline No. of censured patients & 52 & 6 & 46 \\
\hline No. of non-censured patients & 144 & 24 & 120 \\
\hline Mean overall survival, months (range) & $35(0-134)$ & $28(0-102)$ & $36(0-134)$ \\
\hline No. of censured patients & 110 & 19 & 91 \\
\hline No. of non-censured patients & 86 & 11 & 75 \\
\hline
\end{tabular}

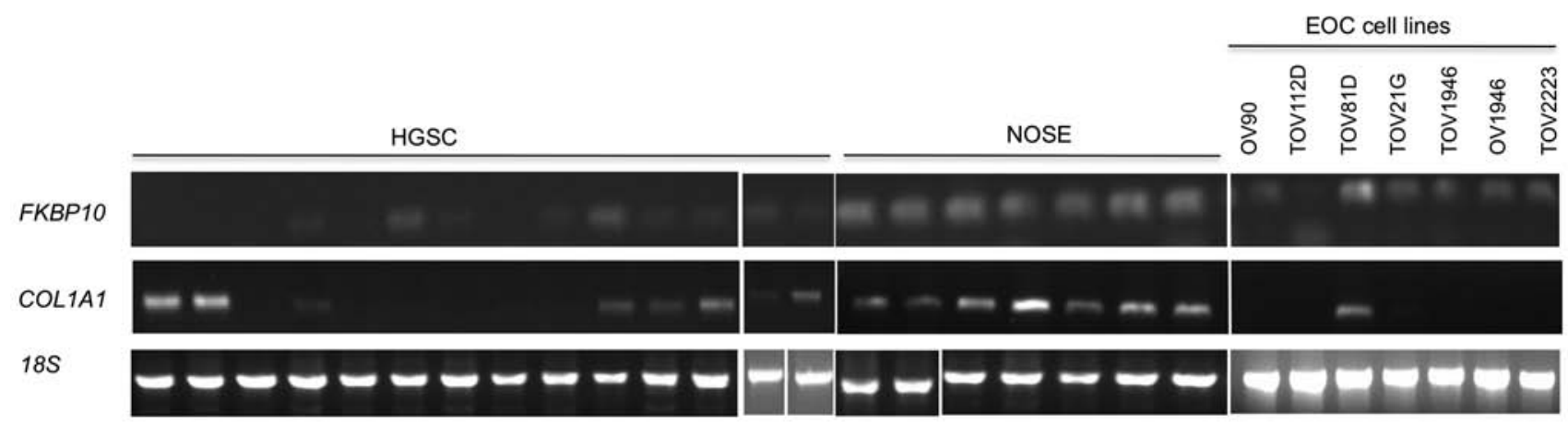

Figure 1. Semi-quantitative RT-PCR analyses of FKBP10 and COL1A1. RT-PCR analyses of FKBP10 and COL1A1 in high-grade serous ovarian cancer samples (HGSC), normal ovarian surface epithelial cells (NOSE) and epithelial ovarian cancer (EOC) cell lines. The RT-PCR expression analysis of $18 \mathrm{~S}$ is also shown for RNA quality.

COL1A1. The inter-observer correlation coefficient was calculated using SPSS software version 16.0 (SPSS Inc., Chicago, IL, USA), where the minimum threshold was 0.7 .

Statistical analyses. Spearman analysis was used to evaluate the correlation of FKBP65 and COL1A1 staining intensities. The association between tumour grade and staining intensity was evaluated using an independent sample t-test. The association between disease stage and staining intensity was evaluated using a one-way ANOVA. The relationship between staining intensity and disease-free interval or overall survival was evaluated using the log-rank test and visualized by Kaplan-Meier survival curve analysis. All statistical analyses were performed with SPSS software version 16.0 (SPSS Inc.), p-values $<0.05$ were considered significant.

\section{Results}

Gene expression analyses of FKBP10 and COL1A1. A previous gene expression microarray analysis of chromosome 17 genes identified $F K B P 10$ and COL1A 1 among the 158 genes underexpressed in HGSCs as compared with NOSEs
(14). To verify these observations, we investigated the expression of these genes by performing RT-PCR analyses on the samples used in the microarray analyses. As shown in Fig. 1, FKBP10 and COLIA1 expression was clearly detectable in all NOSEs. In contrast, FKBP10 expression in the HGSCs was undetectable or expressed at levels lower than that observed in the NOSEs. COL1A1 expression was more variable, ranging from clearly detectable levels comparable to those observed in NOSEs in some samples, to undetectable or lower levels of expression relative to the NOSEs (Fig. 1). Gene expression of FKBP10 and COL1Al was also investigated in cell lines established as long-term passages from chemotherapy naïve EOC samples $(1,26)$. FKBP10 expression was detectable in all EOC cell lines with the highest level of expression observed in TOV81D (Fig. 1). In contrast, COL1A1 expression was detectable in only TOV81D (Fig. 1), which is a non-tumourigenic EOC cell line.

Immunohistochemical staining of FKBP65 and COL1A1. Immunohistochemistry analysis was performed to characterize FKBP65 protein expression in normal ovary and fallopian tube as the expression profile in human tissues purported to 

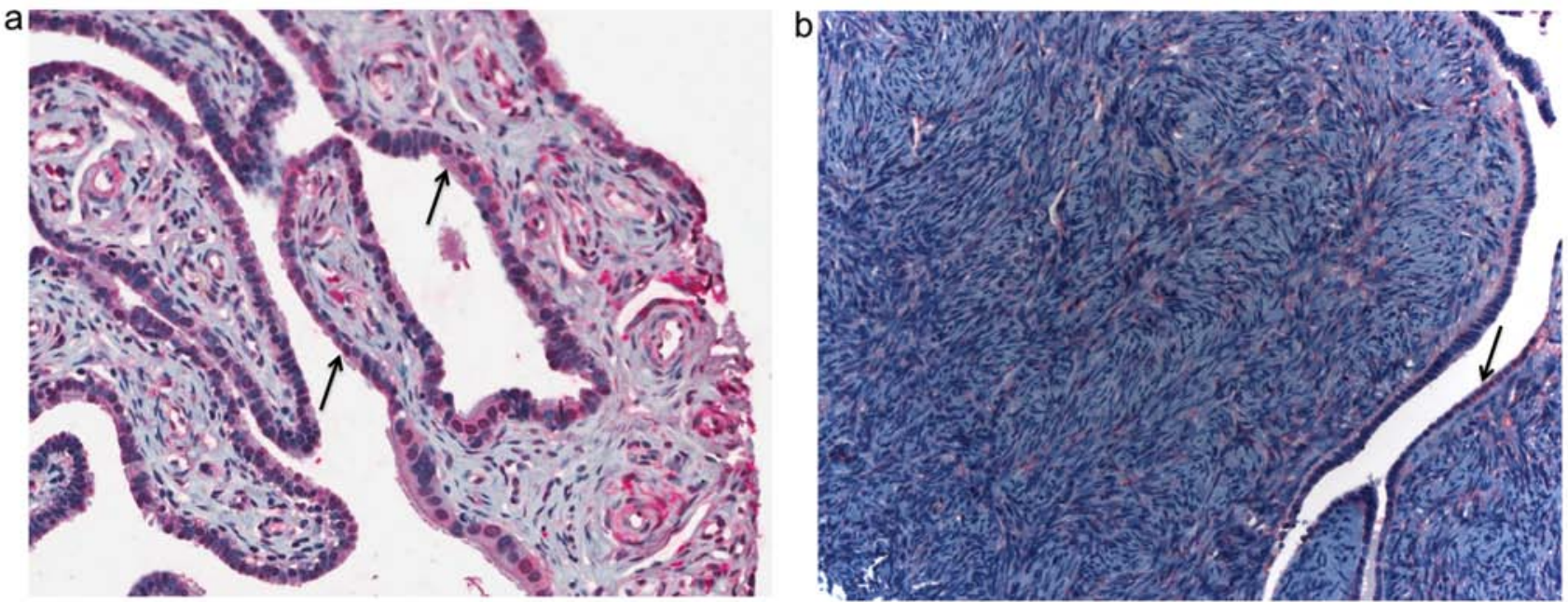

Figure 2. Example of immunostaining of FKBP65 in normal tissues. Immunostaining of FKBP65 in (a) normal fallopian tube tissue and (b) normal whole ovary tissue. Note positive immunoreactivity in epithelial cells as indicated by arrows. Image is shown at x20 magnification from the Aperio image scope scan.

FKBP65

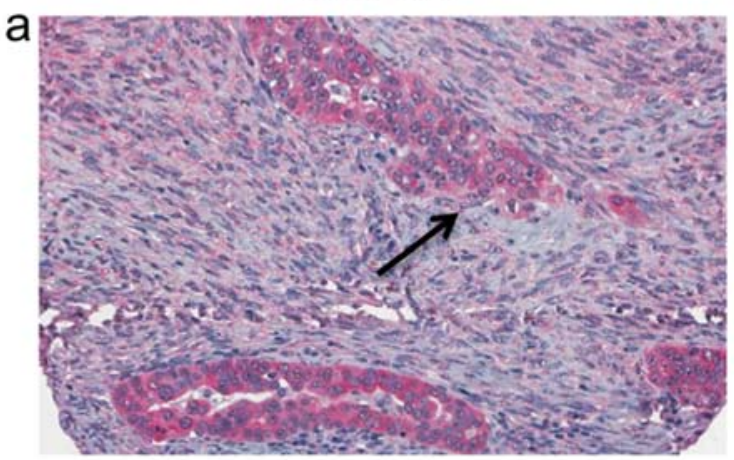

C

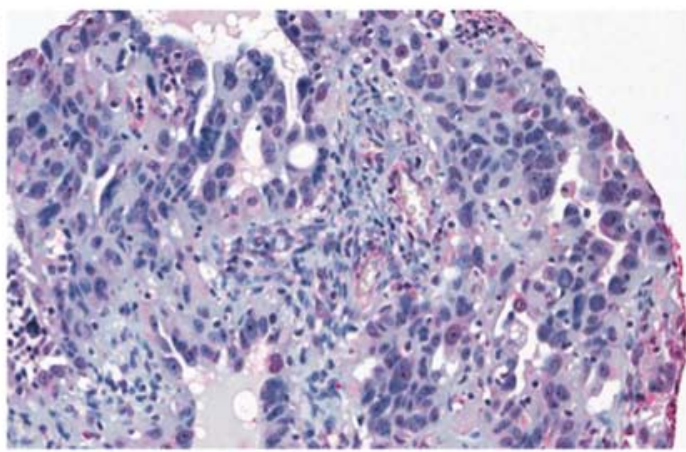

COL1A1
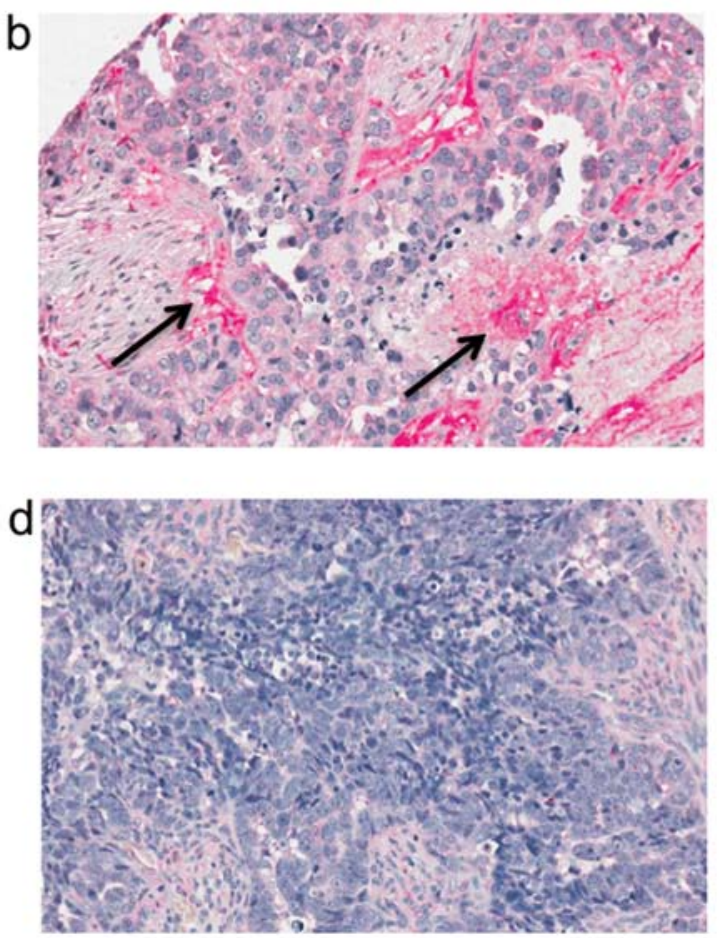

Figure 3. Examples of immunostaining patterns of FKBP65 and COL1A1. Matched HGSC patient cores are (a) and (b), and (c) and (d). (a) High epithelial staining for FKBP65, and (c) no detectable staining of FKBP65. COL1A1 staining is shown in (b) and (d). Arrows indicate positively staining epithelial cells in (a) and positively staining stromal cells in (b). All images are shown at x20 magnification from the Aperio image scope scan.

be the origins of HGSCs have not previously been described. Staining was evident in both the epithelial and stromal cells of the tissues (Fig. 2).

FKBP65 and COL1A1 protein expression in HGSCs was investigated by immunohistochemistry analysis using a tissue array containing 196 cores from tumour samples (Table I). Staining was localized to the cytoplasm for both FKBP65 and COL1A1 and was observed in both the epithelial and stromal cell components of the tumour samples (Fig. 3). To characterize the expression pattern, intensity of staining was scored as absent, low, moderate or high for both the epithelial and stromal cell components where possible, as scoring was not possible for some of the samples due to the type of cells present or the quality of core (Fig. 4). The majority of the epithelial cell components of the tumour samples scored as either low or moderate for FKBP65 expression, and less than 
a
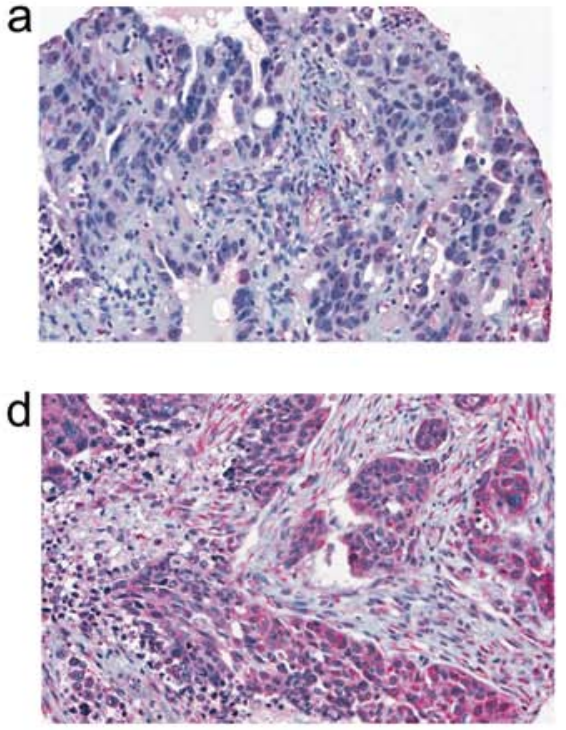

g
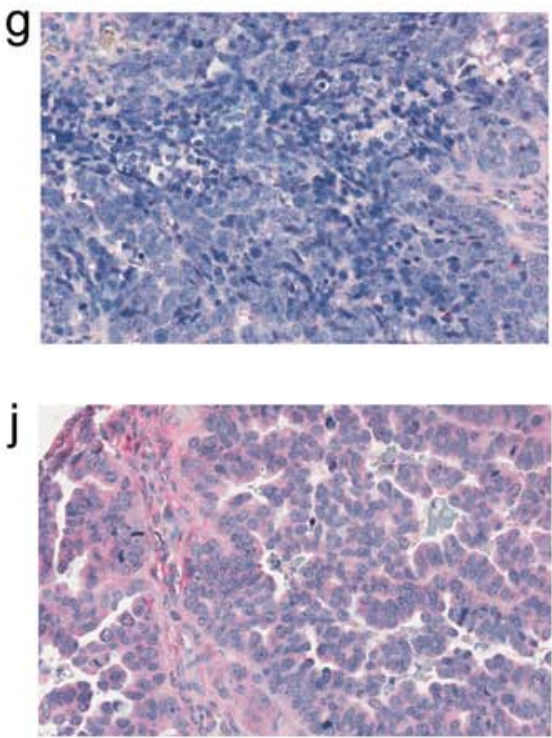
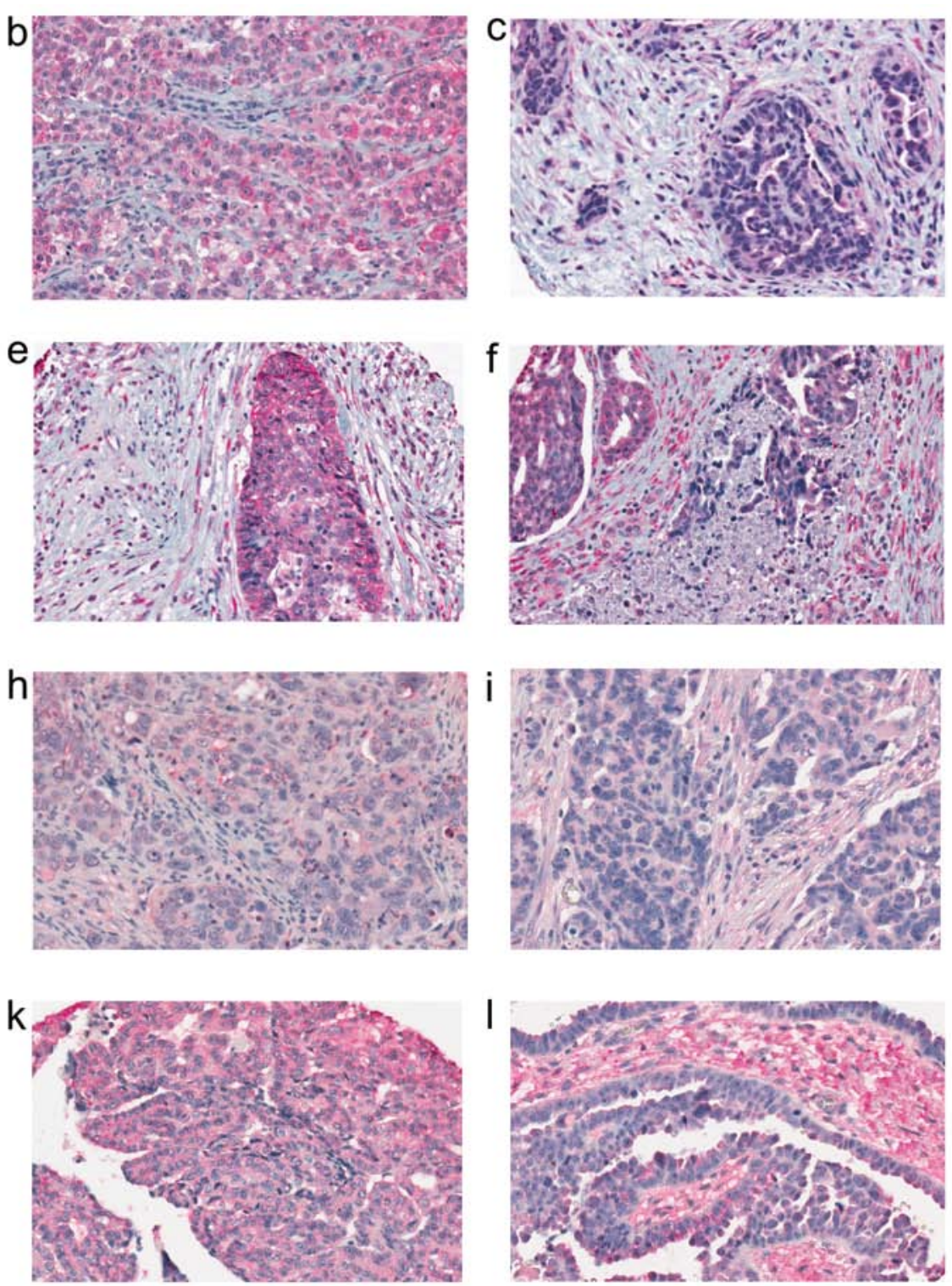

Figure 4. Examples of FKBP65 and COL1A1 staining intensity categories. Examples of FKBP65 staining intensity patterns for (a) absence of epithelial staining, (b) absence of stromal staining, (c) low epithelial and stromal staining, (d) moderate epithelial and stromal staining, (e) high epithelial staining and (f) high stromal staining. Examples of COL1A1 staining intensity patterns for (g) absence of epithelial staining, (h) absence of stromal staining, (i) low epithelial and stromal staining, (j) moderate epithelial and stromal staining, (k) high epithelial staining and (1) high stromal staining. All images are shown at $\mathrm{x} 20 \mathrm{magnification}$ from the Aperio image scope scan.

$10 \%$ of samples scored as either absent or high (Table II). A somewhat similar staining intensity pattern for the epithelial component of the tumours was also observed with COL1A1, although there were more cases that scored with low intensity levels (Table II). In contrast, FKBP65 expression patterns for the stromal components of the tumour were almost equally distributed among all staining intensity categories, with the largest number of samples $(32.3 \%)$ exhibiting moderate staining intensity (Table II). Although the largest number of samples (44.5\%) also exhibited moderate staining of COL1A1 in the stromal component of the tumour, there were fewer samples that had an absent intensity score (Table II). There was a significant correlation between FKBP65 and COL1A1 staining intensity in the epithelial cell component $(\mathrm{p}<0.001)$, but not in the stromal component $(p=0.101)$ of the tumour samples analysed.
Protein expression and disease stage, disease-free interval or overall survival. The staining intensity patterns of FKBP65 and COL1A1 were characterized with respect to disease stage, though the majority of samples $(83 \%)$ were from cases with advanced stage III/IV disease (Table I). There were no statistically significant differences (data not shown) in the distribution of staining intensity patterns for the proteins assayed for either the epithelial or stromal cell components of the tumour samples and disease stage (Table III).

The staining intensities for FKBP65 and COL1A1 were also evaluated with respect to disease-free interval and overall survival. No significant relationships were observed with staining intensities of COL1A1 for the epithelial or stromal components of the tumour samples and either of these clinical parameters (data not shown). Significant relationships were also not found with staining intensities of FKBP65 for the epithelial 
Table II. Distribution of staining intensities for FKBP65 and COL1A1 in HGSC samples.

\begin{tabular}{llccrcc}
\hline \multirow{2}{*}{ Protein } & \multirow{2}{*}{$\begin{array}{c}\text { Total no. of } \\
\text { samples scored }\end{array}$} & \multicolumn{3}{c}{ Staining intensity score (\%) } \\
\cline { 4 - 7 } & Cell type & Absent & Low & Moderate & High \\
\hline \multirow{2}{*}{ FKBP65 } & Epithelial & 193 & $2(1.0)$ & $107(55.4)$ & $69(35.8)$ & $15(7.8)$ \\
& Stromal & 195 & $46(23.6)$ & $40(20.5)$ & $63(32.3)$ & $46(23.6)$ \\
\multirow{2}{*}{ COL1A1 } & Epithelial & 191 & $2(1.0)$ & $134(70.2)$ & $53(27.7)$ & $2(1.0)$ \\
& Stromal & 191 & $9(4.7)$ & $51(26.7)$ & $85(44.5)$ & $46(24.1)$ \\
\hline
\end{tabular}

Table III. Distribution of staining intensities for FKBP65 and COL1A1 by stage.

\begin{tabular}{|c|c|c|c|c|c|c|c|}
\hline \multirow[b]{2}{*}{ Protein } & \multirow[b]{2}{*}{ Cell type } & \multirow[b]{2}{*}{ Stage } & \multirow{2}{*}{$\begin{array}{l}\text { Total no. } \\
\text { of samples } \\
\text { scored }\end{array}$} & \multicolumn{4}{|c|}{ Staining intensity score (\%) } \\
\hline & & & & Absent & Low & Moderate & High \\
\hline \multirow[t]{8}{*}{ FKBP65 } & \multirow[t]{4}{*}{ Epithelial } & I & 15 & 0 & $9(60)$ & $5(33.3)$ & $1(6.6)$ \\
\hline & & II & 18 & 0 & 12 (66.6) & $5(27.7)$ & $1(5.5)$ \\
\hline & & III & 137 & $2(1.5)$ & $75 \quad(54.7)$ & $52(38.0)$ & $8(5.8)$ \\
\hline & & IV & 23 & 0 & 11 (97.8) & $7(30.4)$ & $5(21.7)$ \\
\hline & \multirow[t]{4}{*}{ Stromal } & I & 15 & $5(33.3)$ & $2(13.3)$ & $4(26.6)$ & $4(26.6)$ \\
\hline & & II & 19 & 0 & $6(31.6)$ & $8(42.1)$ & $5(26.3)$ \\
\hline & & III & 138 & $37(26.8)$ & $27(19.6)$ & $42(30.4)$ & $32(23.2)$ \\
\hline & & IV & 23 & $4(17.4)$ & $5(21.7)$ & $9(39.1)$ & $5(21.7)$ \\
\hline \multirow[t]{8}{*}{ COL1A1 } & \multirow[t]{4}{*}{ Epithelial } & I & 15 & 0 & $10(66.6)$ & $5(33.3)$ & 0 \\
\hline & & II & 19 & 0 & $15(78.9)$ & $4(21.1)$ & 0 \\
\hline & & III & 135 & $1(0.7)$ & $98 \quad(72.6)$ & $36(26.7)$ & 0 \\
\hline & & IV & 22 & $1(4.5)$ & $11(50)$ & $8(36.4)$ & $2(9.1)$ \\
\hline & \multirow[t]{4}{*}{ Stromal } & I & 15 & $1(6.6)$ & $2(13.3)$ & $6(40)$ & $6(40)$ \\
\hline & & II & 18 & 0 & 5 (27.8) & 7 (38.9) & $6(33.3)$ \\
\hline & & III & 136 & $6(4.4)$ & $37 \quad(27.2)$ & $65(47.8)$ & $28(20.6)$ \\
\hline & & IV & 22 & $2(9.1)$ & 7 (31.8) & $7(31.8)$ & $6(27.3)$ \\
\hline
\end{tabular}

or stromal components of the tumour samples and disease-free interval (data not shown). In contrast, there was a significant association between prolonged overall survival and the presence of FKBP65 protein in the epithelial component when evaluated for each staining category $(\mathrm{p}=0.005)$ or when cases with no staining are compared with those with low, moderate and high staining combined $(\mathrm{p}<0.001)$ (Fig. 5a and c). Although there are only two samples with no detectable staining in the epithelial component of the tumour, it is interesting that the cases with the highest staining levels for FKBP65 where among the cases which had the longest overall survival (Fig. 5c). A similar analysis of FKBP65 staining found a significant association $(p=0.027)$ with prolonged overall survival when the samples were analysed for the absence or presence of staining in the stromal component (Fig. 5b). Although there was no significant difference with overall survival and staining based on each staining intensity category $(\mathrm{p}=0.087)$, it is interesting that the cases scored absent for staining in the stromal component were among those with the poorest overall survival (Fig. 5d).

\section{Discussion}

In this study we have verified that $F K B P 10$ expression is absent or low in HGSC and the results are consistent with the low frequency of high intensity staining patterns of FKBP65 in tumour samples. FKBP65 expression in the epithelial cells of normal ovarian surface and the distal fimbrae of the fallopian tube is interesting considering that both of these tissues have been proposed as the progenitor cell type for HGSC (34). During the course of this study, decreased expression of FKBP65 in a study of 57 EOC samples of different histological subtypes, which included HGSCs, was reported independently (35). These observations suggest the possibility that FKBP65 expression is also important in the biology of the other histological subtypes of EOC. This notion is supported by the observation that FKBP10 was underexpressed in most of our EOC cell lines, which were derived from epithelial ovarian tumour samples that differed in their histology. 

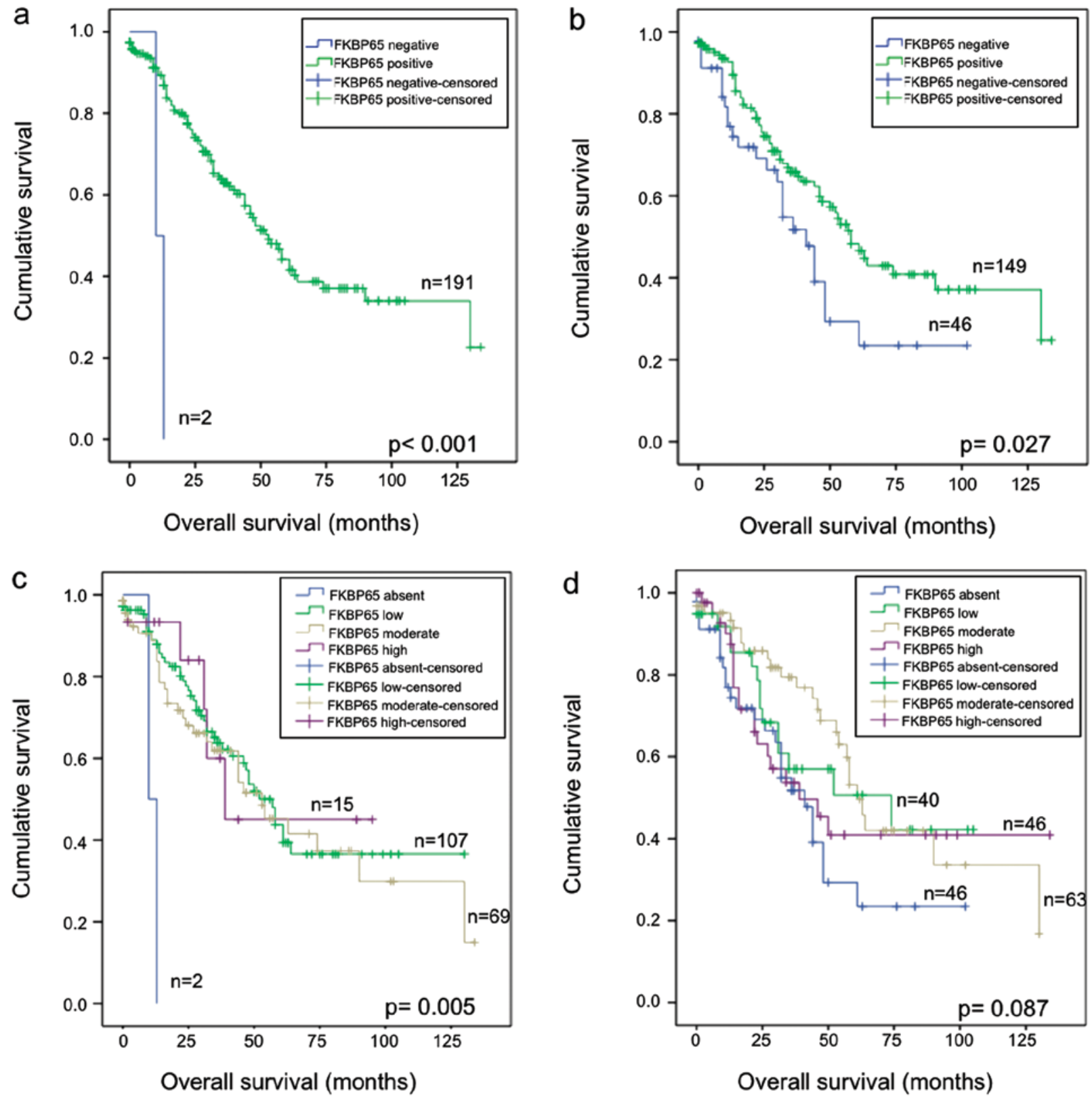

Figure 5. Analysis of FKBP65 staining intensity in HGSCs using the log ratio statistic, and visualized using Kaplan-Meier survival curves. Survival analysis was based on staining intensity grouped as present (positive) or absent (negative) in (a) epithelial and (b) stromal cell components. Survival analysis was based on staining intensity grouped as absent, low, moderate and high in (c) epithelial and (d) stromal cell components.

Our results from the $F K B P 10$ expression assays of the EOC cell lines also suggest that underexpression might be associated with tumourigenicity. Although our assays were semi-quantitative, the highest level of gene expression was observed in TOV81D, the only cell line in our series of EOC cell lines tested that is unable to form tumours in mouse tumour xenograft assays and lacks in vitro growth phenotypes characteristics of tumourigenic cell lines $(26,36)$. Our expression results are consistent with previously published studies from our group where, by semi-quantitative RT-PCR and gene expression microarray analyses, TOV81D exhibited gene expression profiles that resemble those of NOSEs $(3,14,16,37-39)$. Notable also is the underexpression of $F K B P 10$ observed in the tumourigenic OV90 cell line, as this gene was induced in OV90 cell line hybrids that were rendered nontumourigenic as a consequence of the transfer chromosome 3 in genetic complementation assays aimed at identifying genes implicated in tumour suppressor pathways $(15,16)$. Thus, decreased expression of FKBP10 may be important in tumour suppressor pathways.

We observed a significant association between FKBP65 staining intensity in the epithelial cells of HGSC samples and overall survival. An association between high FKBP65 expression and prolonged survival was also observed in the independent study of 57 EOC samples by Henriksen et al, although the finding was not significant (35). In their association analyses the small sample size and possibly the inclusion of different histological subtypes, which are known to exhibit 
differences in outcome (40), may have affected the interpretation of results. In our analyses, though the samples with no FKBP65 staining exhibited the poorest outcome, they represented only two HGSC cases in our cohort. Notable, however, is that the samples exhibiting the highest staining intensity were among the HGSC cases with the longest overall survival. Interestingly, the staining intensities of the stromal component of the tumour samples were also associated with overall survival. There is mounting evidence that ovarian cancer progression involves reciprocal communication between malignant epithelial cells and the adjacent stromal microenvironment possibly relating to malignant epitheliumactivated fibroblasts (41). Although not fully explored in the context of ovarian cancer, gene expression profiles of stromal cells have been found to be predictive of disease outcome in breast cancer (42). The expression profile of COL1A1 was similar to FKBP65 as demonstrated by the significant positive correlation of staining intensities by immunohistochemistry of HGSCs. The similarities in expression profiles have been reported previously, and this is consistent with FKBP65 being a type I collagen chaperone $(19,21)$.

In conclusion, we found FKBP65 underexpressed in a proportion of HGSCs, and its expression correlated with that of COL1A1 in epithelial cells. We also reported on the interesting finding that absence of FKBP65 staining was associated with poorer overall survival warranting replication of our study with larger cohorts. The interesting FKBP10 expression profiles in our EOC cell lines that differ in their tumourigenic potential could also be explored to further elucidate the molecular pathways that involve FKBP65.

\section{Acknowledgements}

We thank Jason Madore for his technical assistance. P.M.W. is a recipient of a Doctoral Research Award (DRA) from the Canadian Institute of Health Research (CIHR). The Research Institute of the McGill University Health Centre and the Centre de Recherche du Centre Hospitalier de l' Université de Montréal receive support from the Fonds de Recherche du Québec - Santé. Clinical specimens were provided by the Banque de tissus et de données of the Réseau de Recherche sur le Cancer of the Fonds de Recherche du Québec - Santé which is affiliated with the Canadian Tumour Repository Network. This research was supported by grants from the CIHR to P.N.T., D.M.P. and A.-M.M.-M.

\section{References}

1. Dion F, Mes-Masson AM, Seymour RJ, Provencher D and Tonin PN: Allelotyping defines minimal imbalance at chromosomal region $17 \mathrm{q} 25$ in non-serous epithelial ovarian cancers. Oncogene 19: 1466-1472, 2000.

2. Foulkes WD, Black DM, Stamp GW, Solomon E and Trowsdale J: Very frequent loss of heterozygosity throughout chromosome 17 in sporadic ovarian carcinoma. Int J Cancer 54: 220-225, 1993.

3. Presneau N, Dewar K, Forgetta V, ProvencherD, Mes-Masson AM and Tonin PN: Loss of heterozygosity and transcriptome analyses of a $1.2 \mathrm{Mb}$ candidate ovarian cancer tumor suppressor locus region at 17q25.1-q25.2. Mol Carcinog 43: 141-154, 2005.

4. Wojnarowicz PM, Provencher DM, Mes-Masson AM and Tonin PN: Chromosome 17q25 genes, RHBDF2 and CYGB, in ovarian cancer. Int J Oncol 40: 18656-1880, 2012.

5. TCGA: Integrated genomic analyses of ovarian carcinoma. Nature 474: 609-615, 2011.
6. Gorringe KL, George J, Anglesio MS, et al: Copy number analysis identifies novel interactions between genomic loci in ovarian cancer. PloS One 5: e11408, 2010.

7. Pieretti M, Cavalieri C, Conway PS, Gallion HH, Powell DE and Turker MS: Genetic alterations distinguish different types of ovarian tumors. Int J Cancer 64: 434-440, 1995.

8. Sangha N, Wu R, Kuick R, et al: Neurofibromin 1 (NF1) defects are common in human ovarian serous carcinomas and co-occur with TP53 mutations. Neoplasia 10: 1362-1372, 2008.

9. Feng Q, Deftereos G, Hawes SE, et al: DNA hypermethylation, Her-2/neu overexpression and p53 mutations in ovarian carcinoma. Gynecol Oncol 111: 320-329, 2008.

10. Rathi A, Virmani AK, Schorge JO, et al: Methylation profiles of sporadic ovarian tumors and nonmalignant ovaries from high-risk women. Clin Cancer Res 8: 3324-3331, 2002.

11. Pergolizzi R, Appierto V, Crosti M, et al: Role of retinoic acid receptor overexpression in sensitivity to fenretinide and tumorigenicity of human ovarian carcinoma cells. Int J Cancer 81: 829-834, 1999.

12. Bruening W, Prowse AH, Schultz DC, Holgado-Madruga M, Wong A and Godwin AK: Expression of OVCA1, a candidate tumor suppressor, is reduced in tumors and inhibits growth of ovarian cancer cells. Cancer Res 59: 4973-4983, 1999.

13. Presneau N, Mes-Masson AM, Ge B, Provencher D, Hudson TJ and Tonin PN: Patterns of expression of chromosome 17 genes in primary cultures of normal ovarian surface epithelia and epithelial ovarian cancer cell lines. Oncogene 22: 1568-1579, 2003.

14. Wojnarowicz PM, Breznan A, Arcand SL, et al: Construction of a chromosome 17 transcriptome in serous ovarian cancer identifies differentially expressed genes. Int J Gynecol Cancer 18: 963-975, 2008.

15. Cody NA, Ouellet V, Manderson EN, et al: Transfer of chromosome 3 fragments suppresses tumorigenicity of an ovarian cancer cell line monoallelic for chromosome 3p. Oncogene 26 : 618-632, 2007.

16. Quinn MC, Filali-Mouhim A, Provencher DM, Mes-Masson AM and Tonin PN: Reprogramming of the transcriptome in a novel chromosome 3 transfer tumor suppressor ovarian cancer cell line model affected molecular networks that are characteristic of ovarian cancer. Mol Carcinog 48: 648-661, 2009.

17. Coss MC, Winterstein D, Sowder RC II and Simek SL: Molecular cloning, DNA sequence analysis, and biochemical characterization of a novel 65-kDa FK506-binding protein (FKBP65). J Biol Chem 270: 29336-29341, 1995.

18. Zeng B, MacDonald JR, Bann JG, et al: Chicken FK506-binding protein, FKBP65, a member of the FKBP family of peptidylprolyl cis-trans isomerases, is only partially inhibited by FK506. Biochem J 330: 109-114, 1998.

19. Patterson CE, Abrams WR, Wolter NE, Rosenbloom J and Davis EC: Developmental regulation and coordinate reexpression of FKBP65 with extracellular matrix proteins after lung injury suggest a specialized function for this endoplasmic reticulum immunophilin. Cell Stress Chaperones 10: 285-295, 2005.

20. Patterson CE, Schaub T, Coleman EJ and Davis EC: Developmental regulation of FKBP65. An ER-localized extracellular matrix binding-protein. Mol Biol Cell 11: 3925-3935, 2000.

21. Ishikawa Y, Vranka J, Wirz J, Nagata K and Bachinger HP: The rough endoplasmic reticulum-resident FK506-binding protein FKBP65 is a molecular chaperone that interacts with collagens. J Biol Chem 283: 31584-31590, 2008.

22. Forbes SA, Bindal N, Bamford S, et al: COSMIC: mining complete cancer genomes in the Catalogue of Somatic Mutations in Cancer. Nucleic Acid Res 39: D945-D950, 2011.

23. Alanay Y, Avaygan H, Camacho N, et al: Mutations in the gene encoding the RER protein FKBP65 cause autosomal-recessive osteogenesis imperfecta. Am J Hum Genet 86: 551-559, 2010.

24. Kelley BP, Malfait F, Bonafe L, et al: Mutations in FKBP10 cause recessive osteogenesis imperfecta and Bruck syndrome. J Bone Miner Res 26: 666-672, 2011.

25. Nishida T, Oda T, Sugiyama T, Izumi S and Yakushiji M: Concurrent ovarian serous carcinoma and osteogenesis imperfecta. Arch Gynecol Obstet 253: 153-156, 1993.

26. Ouellet V, Zietarska M, Portelance L, et al: Characterization of three new serous epithelial ovarian cancer cell lines. BMC Cancer 8: 152, 2008.

27. Le Page C, Marineau A, Bonza PK, et al: BTN3A2 expression in epithelial ovarian cancer is associated with higher tumor infiltrating T cells and a better prognosis. PloS One 7: e38541, 2012. 
28. Therasse P, Arbuck SG, Eisenhauer EA, et al: New guidelines to evaluate the response to treatment in solid tumors. European Organization for Research and Treatment of Cancer, National Cancer Institute of the United States, National Cancer Institute of Canada. J Natl Cancer Inst 92: 205-216, 2000.

29. Ouellet V, Provencher DM, Maugard CM, et al: Discrimination between serous low malignant potential and invasive epithelial ovarian tumors using molecular profiling. Oncogene 24 4672-4687, 2005.

30. Rozen S and Skaletsky H: Primer3 on the WWW for general users and for biologist programmers. Methods Mol Biol 132: 365-386, 2000

31. Karolchik D, Baertsch R, Diekhans M, et al: The UCSC Genome Browser Database. Nucleic Acid Res 31: 51-54, 2003.

32. Arcand SL, Maugard CM, Ghadirian P, et al: Germline TP53 mutations in BRCA1 and BRCA2 mutation-negative French Canadian breast cancer families. Breast Cancer Res Treat 108 399-408, 2008.

33. Davis EC, Broekelmann TJ, Ozawa Y and Mecham RP: Identification of tropoelastin as a ligand for the $65-\mathrm{kD}$ FK506-binding protein, FKBP65, in the secretory pathway. J Cell Biol 140: 295-303, 1998.

34. Salvador S, Gilks B, Kobel M,Huntsman D, Rosen B and Miller D: The fallopian tube: primary site of most pelvic high-grade serous carcinomas. Int J Gynecol Cancer 19: 58-64, 2009

35. Henriksen R, Sorensen FB, Orntoft TF and BirkenkampDemtroder K: Expression of FK506 binding protein 65 (FKBP65) is decreased in epithelial ovarian cancer cells compared to benign tumor cells and to ovarian epithelium. Tumour Biol 32: 671-676, 2011.
36. Provencher DM, Lounis $\mathrm{H}$, Champoux L, et al: Characterization of four novel epithelial ovarian cancer cell lines. In Vitro Cell Dev Biol Anim 36: 357-361, 2000.

37. Birch AH, Quinn MC, Filali-Mouhim A, Provencher DM, Mes-Masson AM and Tonin PN: Transcriptome analysis of serous ovarian cancers identifies differentially expressed chromosome 3 genes. Mol Carcinog 47: 56-65, 2008.

38. Cody NA, Shen Z, Ripeau JS, et al: Characterization of the 3 p12.3-pcen region associated with tumor suppression in a novel ovarian cancer cell line model genetically modified by chromosome 3 fragment transfer. Mol Carcinog 48: 1077-1092, 2009.

39. Manderson EN, Birch AH, Shen Z, Mes-Masson AM, Provencher D and Tonin PN: Molecular genetic analysis of a cell adhesion molecule with homology to L1CAM, contactin 6 , and contactin 4 candidate chromosome $3 \mathrm{p} 26 \mathrm{pter}$ tumor suppressor genes in ovarian cancer. Int J Gynecol Cancer 19: 513-525, 2009.

40. Kobel M, Kalloger SE, Boyd N, et al: Ovarian carcinoma subtypes are different diseases: implications for biomarker studies. PLoS Med 5: e232, 2008.

41. Schauer IG, Sood AK, Mok S and Liu J: Cancer-associated fibroblasts and their putative role in potentiating the initiation and development of epithelial ovarian cancer. Neoplasia 13: 393-405, 2011.

42. Finak G, Bertos N, Pepin F, et al: Stromal gene expression predicts clinical outcome in breast cancer. Nat Med 14: 518-527, 2008. 